\title{
Microcapsule Buckling Triggered by Compression-Induced Interfacial Phase Change
}

\author{
Andrew R. Salmon, ${ }^{\dagger}$ Richard M. Parker, ${ }^{\dagger}$ Alexander S. Groombridge, ${ }^{\dagger}$ Armando Maestro, $^{\dagger}$ \\ Roger J. Coulston, ${ }^{\dagger}$ Jonas Hegemann, ${ }^{\dagger}$ Jan Kierfeld, ${ }^{\dagger}$ Oren A. Scherman, ${ }^{\dagger}$ and Chris Abell ${ }^{*}{ }^{\dagger}$ \\ ${ }^{\dagger}$ Department of Chemistry, University of Cambridge, Lensfield Road, Cambridge CB2 1EW, United Kingdom \\ ${ }^{\ddagger}$ Physics Department, TU Dortmund University, Otto-Hahn-Str 4, Dortmund 44221, Germany
}

\section{Supporting Information}

ABSTRACT: There is an emerging trend toward the fabrication of microcapsules at liquid interfaces. In order to control the parameters of such capsules, the interfacial processes governing their formation must be understood. Here, poly(vinyl alcohol) films are assembled at the interface of water-in-oil microfluidic droplets. The polymer is crosslinked using cucurbit [8] uril ternary supramolecular complexes. It is shown that compression-induced phase change causes the onset of buckling in the interfacial film. On evaporative compression, the interfacial film both increases in density and thickens, until it reaches a critical density and a phase change occurs. We show that this increase in density can be simply

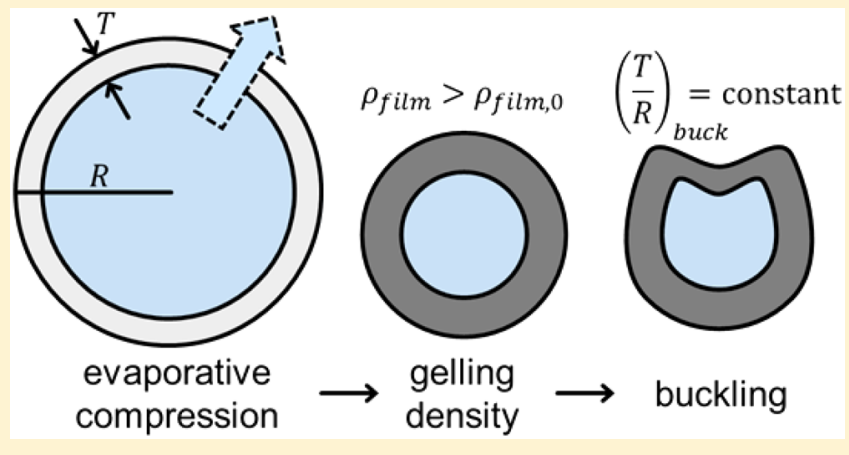
related to the film Poisson ratio and area compression. This description captures fundamentals of many compressive interfacial phase changes and can also explain the observation of a fixed thickness-to-radius ratio at buckling, $\left(\frac{T}{R}\right)_{\text {buck }}$.

$\mathrm{M}$ icroencapsulation is used in some of the world's largest industries. These include applications in cosmetics, ${ }^{1}$ pharmaceuticals ${ }^{2}$ and food, and biotechnology. ${ }^{3}$ Consequently, there are many approaches to fabricating microcapsules, and the development of new materials and superior methodologies that add functionality are of substantial commercial interest. ${ }^{4}$ Many of the oldest and most widely used methods of microencapsulation, including pan coating and air-suspension coating, involve applying a layer of material to solid particles. However, there is an emerging trend toward self-assembling capsules at liquid interfaces, either those of emulsions or liquidin-air droplets. ${ }^{5,6}$ This interest is underpinned both by the increasing development and application of microdroplet-based microfluidics, ${ }^{7}$ and an inherent compatibility with large-scale microcapsule manufacture by spray drying ${ }^{8}$ or high-throughput microfluidic methods. ${ }^{9}$

Recently, Zhang et al. demonstrated a new approach to preparing microcapsules. ${ }^{10}$ In that work it was shown that a supramolecular nanocomposite skin could be self-assembled at the interface of microfluidic droplets via a ternary host-guest complex with cucurbit $[8]$ uril $(\mathrm{CB}[8])$. Using this approach, capsule preparation and cargo loading was achieved in a single step and in high yields. Furthermore, the capsule wall was degraded controllably through disruption of the supramolecular cross-links, allowing for the triggered release of encapsulated cargo. While the microcapsule assembly was directed by the propensity of the nanoparticles to assemble at an oil-water droplet interface, subsequently supramolecular polymer-only capsules have been reported, directed either by an interfacialassembly from both phases ${ }^{11}$ or more recently through electrostatic interactions with a charged surfactant. ${ }^{12}$

While the interfacial assembly of supramolecular polymers has led to the development of functional "smart" microcapsules, ${ }^{13}$ an understanding of the mechanism of capsule formation has been elusive, especially the cause of the "buckling transition" where, after a certain degree of droplet evaporation, wrinkling and crumpling is observed at the droplet interface. Understanding this process is crucial if capsule properties are to be controlled. For example, where evaporation plays a role in capsule formation, increasing the initial amount of material per droplet could result in a thicker capsule skin, denser material packing, or a larger capsule diameter. The mechanisms need to be understood in order to tune these parameters for the specific application.

In the literature the buckling of spherical shells has been understood either in terms of the classic elastic buckling criterion ${ }^{14}$ or (for viscoelastic materials) in terms of a capillary pressure-driven phase transition. ${ }^{15}$ However, here it is found that neither of these models applies to explain the onset of buckling. Instead, we propose a related mechanism that can simply account for a phase transition in terms of the interfacial compression. On compression the interfacial layer thickens and

Received: August 13, 2016

Revised: October 3, 2016

Published: October 4, 2016 

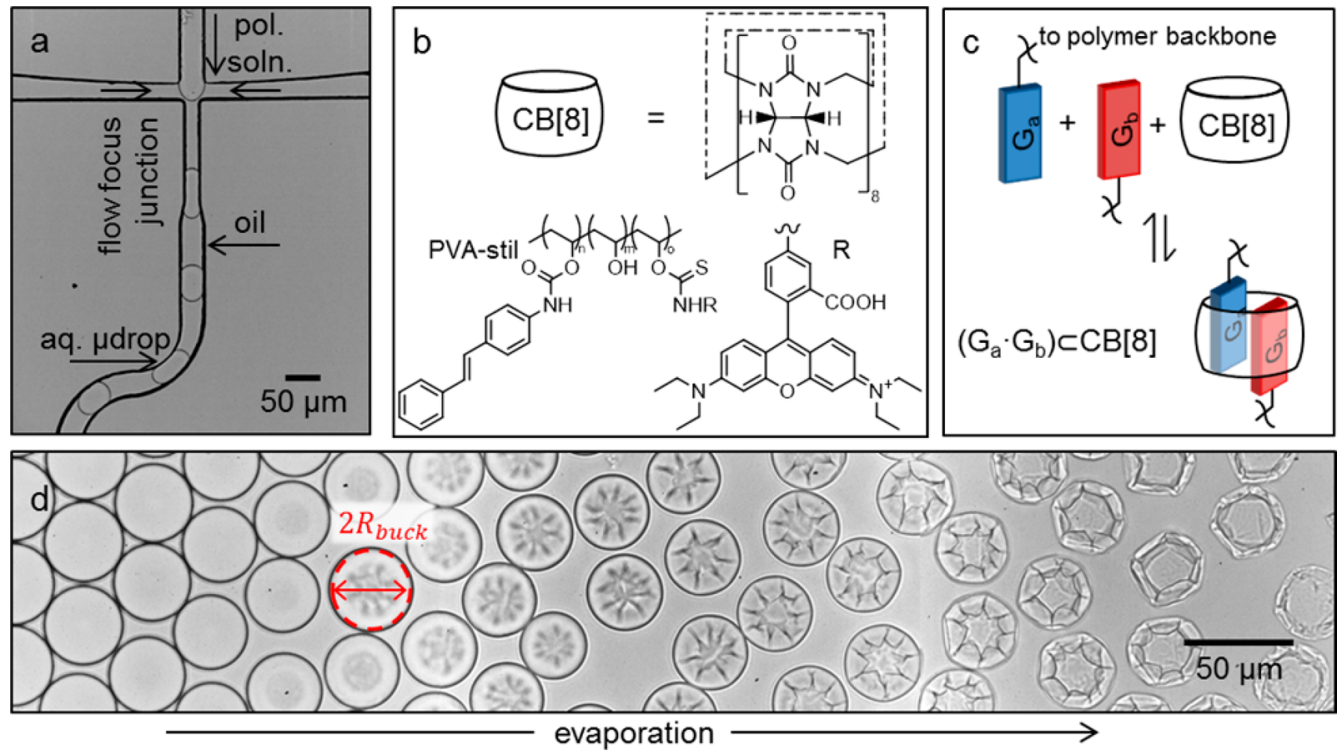

Figure 1. (a) Transmission micrograph of microdroplet formation at a flow-focusing junction. A mixture of polymers and the CB[8] supramolecular cross-linker are loaded into the aqueous microdroplets, which assemble at the water/oil interface to form microcapsules. (b) Molecular structures of the PVA-stil copolymer and the $\mathrm{CB}[8]$ macrocycle. (c) Schematic of the cross-linking supramolecular polymers via CB[8]. (d) Transmission micrograph of the evaporation of microdroplets containing copolymer preassembled at the interface; after a certain decrease in droplet volume a buckling transition is observed.

increases in density. At a critical density this layer undergoes a phase change and becomes elastic. After the material becomes elastic it almost immediately buckles because (in accordance with the classical buckling criterion) the critical change in shell volume is extremely small. Here the phase change is a gelation that is dependent on reaching a sufficient cross-link density; however, the general mechanism captures fundamentals of many compressive phase changes and buckling transitions and so will likely apply widely.

\section{EXPERIMENTAL SECTION}

Materials. Cucurbit[8]uril ( $\mathrm{CB}[8])$ was synthesized from glycoluril and formaldehyde, as previously reported. ${ }^{16,17}$ The functional polymer "PVA-stil" was synthesized by coupling rhodamine B isocyanate and stilbene isocyanate onto a commercial poly(vinyl alcohol) (PVA) backbone (Sigma, Mowiol 6-98, $M_{\mathrm{w}}=47 \mathrm{kDa}$ ) according to a reported procedure. ${ }^{12}$ The rhodamine $\mathrm{B}$ isocyanate gives the polymer a positive charge and allows it to be electrostatically held at the interface. The loading of stilbene was determined to be 0.5 mol \% (i.e., $0.5 \%$ of vinyl alcohol subunits were coupled to a stilbene) by UV-vis spectroscopy.

The capsule-forming aqueous phase was prepared from PVA-stil and $\mathrm{CB}[8]$ such that the $\mathrm{CB}[8]$ :stilbene ratio was $60: 6 \mu \mathrm{M}$. We find this to be the stoichiometric excess of $\mathrm{CB}[8]$ at which the buckling radius saturates (see discussion).

Microfluidics. Poly(dimethylsiloxane) (PDMS) microfluidic devices were prepared by a standard soft lithography procedure. ${ }^{18}$ Briefly, PDMS was cast on to a SU-8 master (made by UV photolithography) and cured at $70{ }^{\circ} \mathrm{C}$ overnight. The PDMS was then peeled from the master, $1.0 \mathrm{~mm}$ holes were punched for the inlets/outlets, and finally the PDMS was plasma bonded to a glass slide to enclose the microchannels. In order to make the channels fluorophilic, they were then functionalized by flowing a freshly prepared solution of trichloro $(1 \mathrm{H}, 1 \mathrm{H}, 2 \mathrm{H}, 2 \mathrm{H}$-perfluorooctyl)silane (Alfa Aesar, $0.5 \% v / v$ in FC40) through the channels, allowing to stand for $5 \mathrm{~min}$, and then flushing through with nitrogen. Flow was driven using syringe pumps (Harvard Apparatus, PHD 2000) with $1.0 \mathrm{~mL}$ plastic syringes (NORM-JECT) using tubing (PORTEX, polyethene $1.09 \mathrm{~mm}$ OD, $0.38 \mathrm{~mm}$ ID) friction fits to connect the devices to the syringes.
For the continuous oil phase, Fluoroinert FC-40 (3M), with 2 wt \% surfactant (XL-01-171, Sphere Fluidics) was employed. To drive capsule formation, 0.5 wt \% carboxylate terminated poly(hexafluoropropylene oxide) (Krytox 157FS-L, DuPont) was added as the negatively charged dopant "krytox ${ }^{(-)}$, except where stated otherwise.

Microscopy. Microscopy was generally performed in transmission with an Olympus IX-71 inverted microscope, equipped with a high frame rate camera (Phantom V72, Vision Research). For monitoring the interfacial fluidity using nanoparticles (Figure 4) an Olympus BX51 equipped with incident geometry dark-field optics was used. Gold nanoparticles were made in three steps according to a reported method $^{19}$ and sized by scanning transmission electron microscopy (Hitachi S-5500), with mean diameters and standard deviations given from a minimum of 50 measurements.

Buckling Radii. Initial and buckling radii were measured from calibrated transmission micrographs by circle fitting, using ImageJ software. The microcapsule buckling diameter was taken at the first signs of droplet asphericity and/or wrinkling. Values are given from a minimum of 10 measurements, with the standard deviation given as the error.

Pendant Droplet Measurements. Pendant droplet measurements were performed using a commercial instrument (First Ten Angstroms, FTA1000). At room temperature, the oil-phase (FC-40, 0.01 wt $\%$ XL-01-171, 0.0017 wt $\%$ krytox $^{(-)}$) was hung from a 20 gauge needle $(0.914 \mathrm{~mm} \mathrm{OD})$ and allowed to equilibrate with the aqueous phase $(6 \mu \mathrm{M}$ stilbene, $60 \mu \mathrm{M} \mathrm{CB})$ for $10 \mathrm{~min}$. For interfacial compression the droplet volumes were reduced from 3 to $0.5 \mu \mathrm{L}$ over $10 \mathrm{~s}$. Buckled droplet elastometry was carried out according to the reported methodology. ${ }^{20}$

\section{RESULTS AND DISCUSSION}

Uniform polymer microcapsules were prepared from monodisperse microdroplets using a similar method to that reported previously. ${ }^{10,12}$ The microdroplets comprise of an aqueous solution of cucurbit[8]uril ( $\mathrm{CB}[8])$ and PVA-stil polymer (Figure $1 \mathrm{~b}$ ), which is based on a commercially available poly(vinyl alcohol) (PVA) backbone. The PVA backbone is modified by the covalent attachment of stilbene side groups as "handles" for supramolecular polymer cross-linking through a 

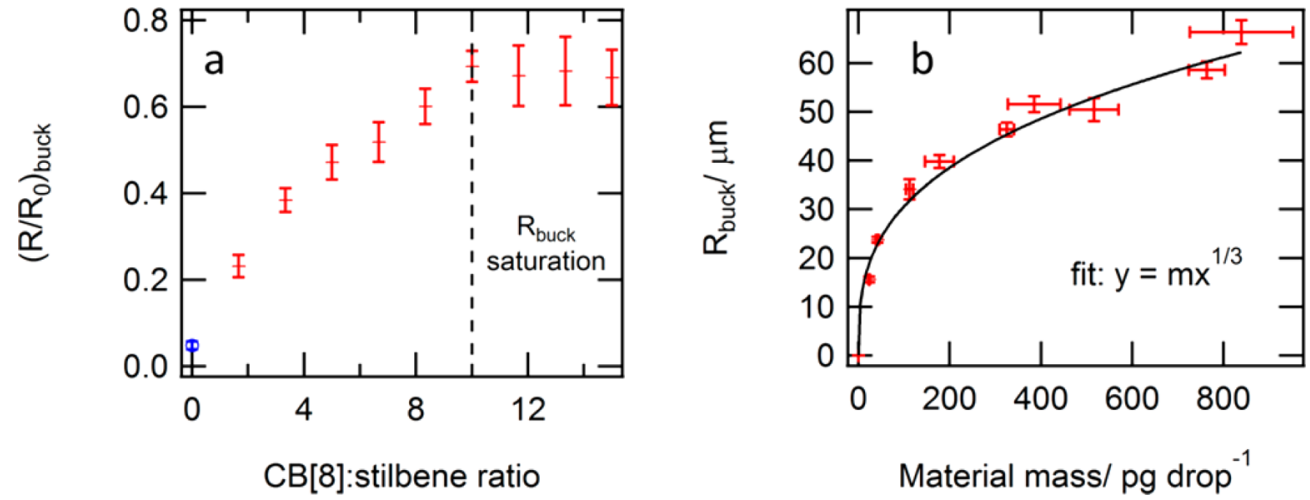

Figure 2. (a) Buckling radius ratio as a function of the $\mathrm{CB}[8]$ :stilbene ratio. For the homoternary (PVA-stil.PVA-stil) $\subset \mathrm{CB}[8]$ complex there are two stilbene moieties for each complex, and so a $\mathrm{CB}[8]$ :stilbene ratio of 0.5 is the stoichiometric ratio. The buckling ratio increases with a stoichiometric excess of $\mathrm{CB}[8]$ until saturating at a ratio of $10: 1$. The blue data point is distinguished because in the absence of $\mathrm{CB}[8]$ "particles" are formed rather than buckled capsule shells. (b) Buckling radius as a function of material mass per droplet. To an excellent fit the radius scales with the inverse cube of the material mass. This implies a constant thickness-to-radius ratio at buckling, $\left(\frac{T}{R}\right)_{\text {buck }}$. A CB $[8]$ :stilbene ratio of 10:1 was used. The material mass is calculated from the concentration and initial droplet diameter.

1:2 homoternary host: guest complex with $\mathrm{CB}[8]$ (Figure 1c). ${ }^{21,22}$

Water-in-oil microdroplets were prepared from this polymer$\mathrm{CB}[8]$ mixture using a PDMS-based flow-focusing microfluidic device (Figure 1a). By ensuring a complementary electrostatic interaction between the polymer matrix in the aqueous phase and charged surfactants in the perfluorinated oil phase, the polymer is localized to the microdroplet interface. ${ }^{12}$ Following formation the microdroplets were directly deposited onto a glass slide. The aqueous microdroplets are less dense than the residual fluorocarbon oil phase so they float and gradually evaporate. After a certain degree of evaporative shrinkage the interfacial polymer film is seen to wrinkle and buckle (Figure 1d). The spherical shell ultimately collapses to give a disc with a thick folded rim (Figure S3). The evaporation gradient is obtained by placing a drop of the emulsion on a glass slide. The microdroplets toward the edge of the patch of emulsion evaporate more quickly. We believe that this evaporation gradient caused by a local humidity effect. The droplet density is lower toward the edge of the emulsion patch, and so the droplets will evaporate to a greater extent before the air is locally saturated.

One approach to analyzing this buckling transition is to vary the concentration of the capsule-forming components and measure the effect on the buckling diameter. For example, if the concentration of the capsule-forming components is increased then it could be expected that the size of the resulting microcapsules would also increase. Similarly, the volume of the initial microdroplets can be controlled through the relative flow rates and differently sized flow-focus junctions. However, by quantitatively assessing the proportionality of this relationship, further insight into the nature of this buckling transition can be ascertained.

The buckling radii, $R_{\text {buck }}$ were systematically measured for microdroplets with initial radii, $R_{0}$, over the range 30-100 $\mu \mathrm{m}$ (Figure $2 b$ ). The $\mathrm{CB}[8]$ :stilbene ratio is such that there is a large stoichiometric excess of $\mathrm{CB}$. Here we use stilbene and $\mathrm{CB}[8]$ concentrations of 6 and $60 \mu \mathrm{M}$, respectively, which we find are the relative concentrations at which the buckling diameter saturates as a function of $\mathrm{CB}[8]$ concentration (Figure $2 \mathrm{a})$. At this ratio the amount of $\mathrm{CB}[8]$ and polymer is similar by mass. In control experiments it is found that $\mathrm{CB}[8]$ does not form capsules independently of the polymer, and so the greater capsule radius is not simply as a result of additional material mass.

$R_{\text {buck }}$ was found to increase with greater concentrations and greater $R_{0}$, but more universally it was found that $R_{\text {buck }}$ depends on the amount of cross-linked polymeric material in the droplet, independent of whether additional mass originates from greater concentration or a larger droplet volume. To an excellent fit, $R_{\text {buck }}$ scales with the inverse cube of the capsule material mass (Figure 2b). This finding is not particular to the polymer system used here, as is confirmed using the heteroternary cross-linked system (PVA-stil·PVA-mv) CCB[8] discussed in previous work (Figure S1). ${ }^{12}$ This scaling implies that the capsule wall thickness is greater for larger capsules. Assuming a constant material density at buckling, then geometrically (assuming the wall thickness is much smaller than the capsule radius, which is shown later) the wall thickness at buckling, $T_{\text {buck }}$ depends on the surface area at buckling, $A_{\text {buck }}$ as

$$
T_{\text {buck }} \propto \frac{m_{\text {cap }}}{A_{\text {buck }}} \propto \frac{m_{\text {cap }}}{R_{\text {buck }}^{2}}
$$

where $m_{\text {cap }}$ is the mass of capsule forming material present within the droplet. As we have seen experimentally, $m_{\text {cap }} \propto$ $R_{\text {buck }}^{3}$ (Figure $2 \mathrm{~b}$ ) and so substituting in we find

$$
T_{\text {buck }} \propto \frac{R_{\text {buck }}^{3}}{R_{\text {buck }}^{2}} \propto R_{\text {buck }}
$$

Notably this implies that the thickness-to-radius ratio at buckling $\left(\frac{T}{R}\right)_{\text {buck }}$ is constant. We additionally confirm a constant ratio $\left(\frac{T}{R}\right)_{\text {buck }}$ by atomic force microscopy and optical interferometry, after collecting the capsules in the dry state (Figure S3, S4). In the dry state $\left(\frac{T}{R}\right)_{\text {buck }} \approx 0.014$. This ratio is consistent with that expected from the number of secondary buckling wrinkles around the primary indent (s-cones). The calculation for eight $s$-cones (Figure 1d) also gives $\left(\frac{T}{R}\right)_{\text {buck }} \approx 0.014 .^{14}$ 


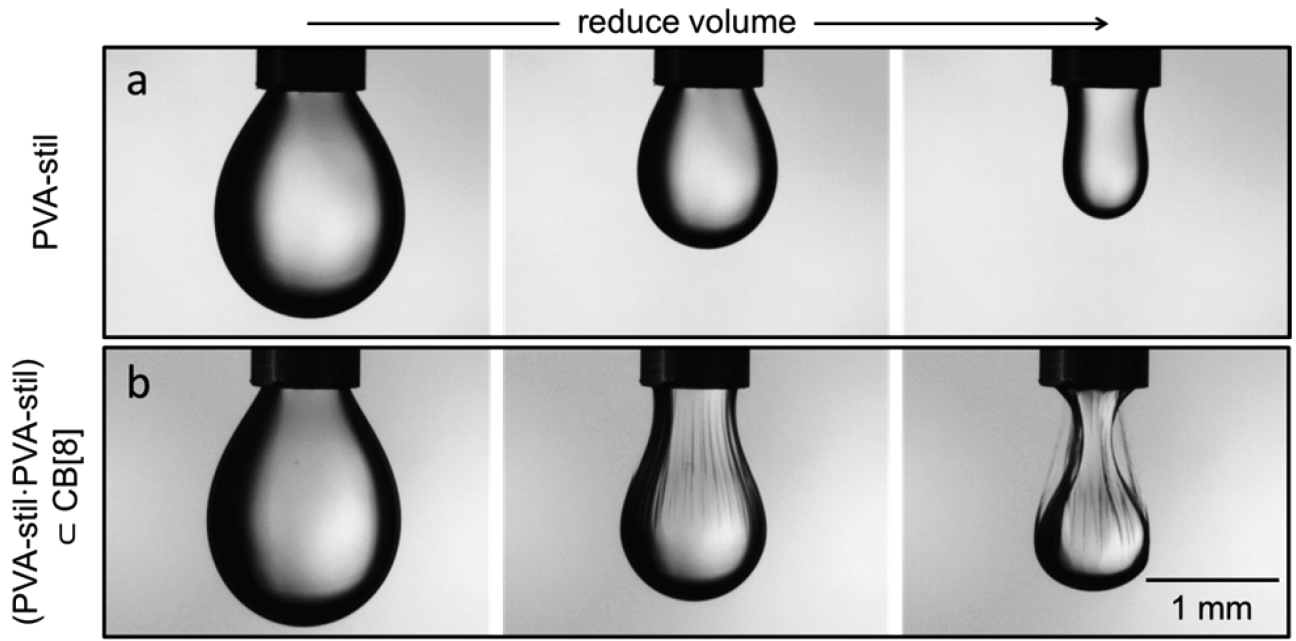

Figure 3. Oil-in-water pendant droplets undergoing interfacial compression by negative pressure in the capillary with (a) PVA-stil localized at the droplet interface and (b) in the presence of the $\mathrm{CB}[8]$ cross-linker. Only with cross-linking is buckling of the interfacial copolymer film observed.

It is noted that in our case a constant ratio $\left(\frac{T}{R}\right)_{\text {buck }}$ also corresponds to a constant fractional area compression $\left(\frac{A_{\text {buck }}}{A_{0}}\right)$. This correspondence follows because $m_{\text {cap }} \propto R_{\text {buck }}^{3} \propto V_{\text {buck }}$ (as above) and $m_{\text {cap }} \propto V_{0}$ (for constant material concentration). However, a constant experimental ratio, $\left(\frac{A_{\text {buck }}}{A_{0}}\right)$, does not in this case imply a constant surface concentration at buckling, $\frac{m_{\text {cap }}}{A_{\text {buck }}}$, because the initial surface concentration is dependent on the initial droplet volume. That is, the buckling is not triggered at a fixed surface concentration.

A constant ratio $\left(\frac{T}{R}\right)_{\text {buck }}$ has been found in several other reports on interfacial shell and capsule buckling. Specifically, this ratio is characteristic of two distinct types of shell buckling:

(1) The buckling of elastic spherical shells at a critical pressure. ${ }^{14,23,24}$ As will be shown, this model is ruled out for our system because the change in shell volume that would induce buckling, $\left(\frac{\Delta V}{V}\right)_{\text {buck }}$, is extremely small which cannot be reconciled with the relatively large changes observed in droplet volume.

(2) Pressure-induced viscous to elastic phase change, followed by elastic buckling. ${ }^{15}$

To assess these mechanisms it is necessary to characterize the elastic properties of the cross-linked polymer film. We used pendant drops as a mimic for the microfluidic droplets, with the denser fluorocarbon phase used to form the droplet, so that the measurements can be performed in the conventional "hanging" geometry. During the experiment, volume was withdrawn from the droplet resulting in the compression of the oil/water interface. With only PVA-stil present at the droplet interface (i.e., in the absence of the $\mathrm{CB}[8]$ cross-linker), a smooth decrease in droplet surface area was observed (Figure 3a). In the presence of $\mathrm{CB}[8]$ the interface was observed to buckle for surface area compressions $\frac{\Delta A}{A_{0}}>0.5$ (Figure $3 b$ ). It is known that conventional dilation measurement procedures fail for buckled interfaces. ${ }^{25}$ However, a method to extract mechanical film parameters from buckled droplet profiles has been reported. $^{20}$ In the same work it is shown that the film thickness, $T$, can be deduced from the wrinkle wavelength. Using this reported procedure, the mechanical properties of the buckled film were calculated (Table 1).

Table 1. Geometric and Mechanical Parameters of a Buckled Pendant Droplet Film (Figure $3 b$, middle) ${ }^{a}$

\begin{tabular}{ll}
\multicolumn{1}{c}{ parameter } & \multicolumn{1}{c}{ value } \\
surface tension, $\gamma$ & $18.7 \pm 0.9 \mathrm{mN} \mathrm{m}^{-1}$ \\
2D Young's modulus, $Y_{2 \mathrm{D}}=Y_{2 \mathrm{D}} T$ & $57.2 \pm 4.5 \mathrm{mN} \mathrm{m}^{-1}$ \\
poisson ratio, $\nu$ & $0.29 \pm 0.03$ \\
wrinkle wavelength & $84 \pm 25 \mu \mathrm{m}$ \\
film thickness, $T$ & $320 \pm 190 \mathrm{~nm}$
\end{tabular}

${ }^{a}$ Parameters were derived using the reported pendant shape analysis. $^{20}$.

With the assumption that the interfacial film is similar between the microfluidic and pendant droplet cases, we can now use these mechanical properties to assess the first of the reported buckling mechanisms. The buckling criterion for elastic spherical shells is well-known. There is a critical pressure difference across the shell wall at which point the shell will buckle, $\Delta P_{\text {buck, }}$ that is given by ${ }^{14}$

$$
\Delta P_{\text {buck }}=\frac{2 Y_{3 \mathrm{D}}}{\sqrt{3\left(1-\nu^{2}\right)}}\left(\frac{T}{R}\right)_{\text {buck }}^{2}
$$

where $Y_{3 \mathrm{D}}$ is the 3-dimensional Young's modulus and $\nu$ is the Poisson ratio. Thus, in this model the constant ratio $\left(\frac{T}{R}\right)_{\text {buck }}$ that we measure would correspond to a constant pressure at buckling. The classic buckling criterion can also be expressed in terms of the volume change required to cause buckling. On deflation an elastic shell will initially undergo an isotropic ("spherical") deformation until: ${ }^{14}$

$$
\left(\frac{\Delta V}{V}\right)_{\text {buck }}=\frac{T}{R} \sqrt{3\left(\frac{1-\nu}{1+\nu}\right)}
$$

where $V$ is the shell volume. With the Poisson ratio and film thickness measured here (Table 1) a shell volume reduction, $\left(\frac{\Delta V}{V}\right)_{\text {buck }}$, of order $10^{-3}$ would be sufficient to trigger buckling if 


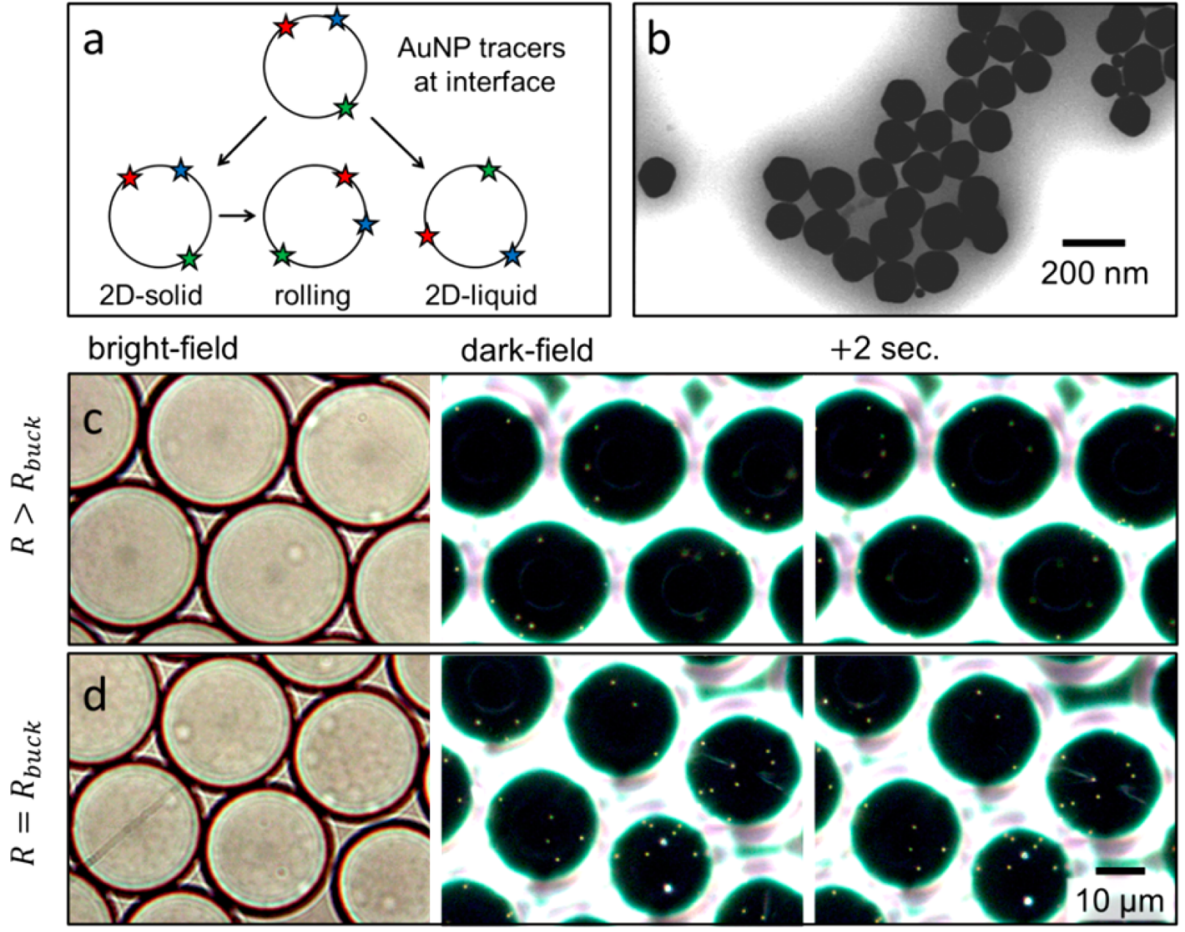

Figure 4. (a) Schematic of the use of AuNPs to monitor the fluidity of the droplet interface. (b) Scanning transmission electron micrograph of the AuNPs used. (c, d) Micrographs of microdroplets with an interfacial copolymer layer containing AuNP-tracers: (c) before and (d) after the buckling transition. The AuNP tracers are seen as bright points in the dark-field micrographs. With $R>R_{\text {buck }}$ the interface is liquid, and at $R=R_{\text {buck }}$ it transitions to solid. Relatively large AuNPs $(\varnothing=138 \pm 5 \mathrm{~nm})$ are used so that their scattering can be easily visualized in dark-field microscopy.

the film was behaving elastically. With the large areacompressions we observe before buckling $\left(\frac{\Delta A}{A_{0}}>0.5\right)$ such a small change in shell volume would require the film to deform without substantial volume-compression, and this requires a Poisson ratio close to 0.5 . This is not in accordance with the measured Poisson ratio of 0.29 , implying that the onset of buckling cannot be explained by the theory for elastic shells.

In the work of Tsapis et al. the buckling of evaporating drops containing colloidal (polystyrene) nanoparticles was described. ${ }^{15}$ It was found that while the droplets ultimately buckled analogous to an elastic shell, the onset of buckling could not be explained by the classic elastic buckling criteria. Instead a viscoelastic model was proposed, with the shell initially viscously yielding under capillary pressure, until a phase change triggered elastic behavior. This phase change was associated with a pressure drop across the shell wall, with a critical pressure overcoming the electrostatic repulsion between particles, "jamming" the film, and causing the phase change to an elastic solid.

In order to assess if the onset of buckling in this work was caused by a phase change, a small quantity of gold nanoparticles (AuNPs) was added to the aqueous phase. The citratestabilized (negatively charged) AuNPs attach to the positively charged polymer and allow for the mobility of the interface to be tracked by dark-field microscopy (Figure 4). If the interface is fluid there should be no correlation between the relative AuNP positions over time. However, if their relative positions are correlated, the shell is either very viscous or solid. Before the buckling transition (i.e., $R>R_{\text {buck }}$ ) the interface exhibited fluid behavior (Figure 4c), with the relative position of individual AuNPs changing in time. Then, after a certain degree of shrinkage, a phase change was observed with the nanoparticle diffusion/convection stopping, indicating the interface becoming solid. This transition occurred just before droplet wrinkling and the onset of asphericity was observed (Figure 4d).

The clear implication is that the capsule buckling transition is triggered by a fluid-to-solid phase transition in the polymer matrix at the interface. Initially the film undergoes a viscous deformation, with the film yielding and thickening. Once the film becomes solid it behaves elastically and the elastic buckling criterion applies. The phase change and buckling of the interfacial shell are almost coincident because the critical change in volume, $\left(\frac{\Delta V}{V}\right)_{\text {buck }}$, is extremely small (of order $10^{-3}$, as above).

Having shown that the buckling transition is associated with a phase change, this leaves the question of what the precise cause of the phase change is, and why it occurs at a critical ratio $T / R$. According to Tsapis et al. the pressure drop across the shell at an aqueous-air interface was expressed using Darcy's law:

$$
\Delta P=\eta T J / k
$$

with fluid viscosity $\eta$, shell permeability $k$, and volumetric flux across the shell $J$; expressed as the change in radius $\mathrm{d} R$ over time $\mathrm{d} t$ ). That is, as the shell becomes thicker it takes a greater pressure differential to drive the fluid across the interface (for evaporation) at a given volumetric flux. The pressure drop at buckling was found to be a constant when varying the volume fraction of nanoparticles (i.e., the concentration) and the initial drop size, and was associated with the pressure required to overcome the repulsive potential between particles and to "jam" the interface. Using mass conservation to determine the shell thickness, and the Carmen-Kozeny relation to determine the 
shell permeability it was found that this critical pressure is equivalent to a critical ratio $T / R$.

However, the model used in the work by Tsapis et al. makes predictions that are not borne out experimentally here. First, in the case of the pendant droplet experiments there is no evaporative flux yet the same buckling behavior is observed. Second, the pressure on the capsule membrane is expected to be proportional to the volumetric flux. However, when we adjust the volumetric flux (by a factor of $\sim 6$ ) by increasing the rate of evaporation we observe no change in the buckling diameter (Table S1). While the presence of a phase transition and subsequent elastic behavior is confirmed, the microscopic model of the phase transition proposed by Tsapis et al. does not seem to apply to the interfacial polymer films considered here. This is perhaps not surprising as for particle shells the phase transition is jamming, whereas here the phase transition is a gelation.

Instead we propose that the phase change is caused by the concentration of the capsule forming material on compression of the interface. The first stage of interfacial compression would be expected to involve the exclusion of surfactant from the interface. If this were the case, then it would be anticipated that the buckling diameter would be dependent on the surfactant concentration. If the surfactant concentration is higher, then a larger interfacial compression would be required to first expel the surfactant, and the buckling diameter would be smaller. Such a trend is confirmed by experiment (Figure 5); thus, there is evidence for an initial deformation regime where the interfacial layer compresses without thickening $(\nu=0)$.

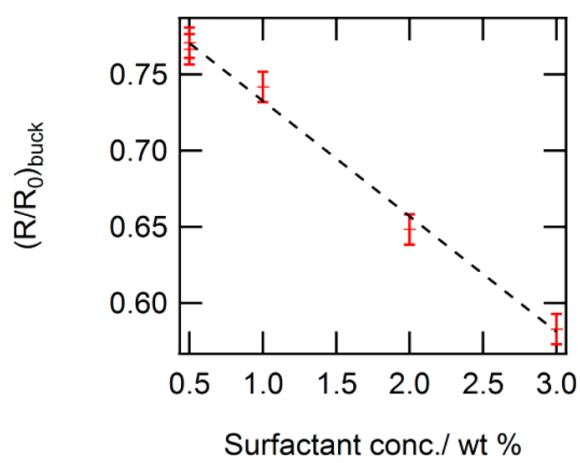

Figure 5. Buckling ratio as a function of oil surfactant concentration. Aqueous: $[\mathrm{CB}[8]$ :stilbene $]=60: 6 \mu \mathrm{M}$. Oil: FC40, 3-0.5:0.5-0.08 wt $\%$ XL1171: krytox $^{(-)}$, in proportion.

However, if compressive exclusion of the surfactant was the only factor then it would not be clear why a constant ratio $(T /$ $R)_{\text {buck }}$ is implied here (Figure $2 b$ ). It would be expected that the polymer mass would directly relate to the shell surface area, when instead it relates experimentally to the enclosed shell volume. Hence the mechanism must involve viscoelastic deformation to thicken the interfacial film during compression. If concentration of capsule-forming material is the cause of the phase change, then the process of thickening of the film must also increase its density.

An increase in film density on compression is implied by any Poisson ratio $0<\nu<0.5$. Specifically, it follows from the definition of the Poisson ratio that for a shrinking spherical shell the film density, $\rho_{\text {film }}$ is given by (see the Supporting Information for derivation):

$$
\rho_{\text {film }}=\rho_{\text {film }, 0}\left(\frac{R_{0}}{R}\right)^{2-4 \nu}
$$

That is, for any Poisson ratio $0<\nu<0.5$, as the droplet shrinks the film thickens but also increases in density. It has been confirmed previously that interfacial films that undergo similar phase change behavior do indeed thicken under compression. ${ }^{26}$ In the same work it was shown that the thickness of the interfacial film increases approximately linearly with the surface concentration, implying a constant Poisson ratio, but does not increase in proportion to the surface concentration, implying an increase in the film density. Thus, we have a strong basis to assume a constant Poisson ratio $0<\nu$ $<0.5$. We also measure a Poisson ratio of 0.29 after the phase change (Table 1). With these assumptions, if there is a critical film density where a phase change occurs, then a phase change will occur at a critical radius ratio $R_{0} / R_{\text {buck. As already }}$ mentioned, for a given film density the film thickness, $T_{\text {buck }}$ has the proportionality:

$$
T_{\text {buck }} \propto \frac{m_{\text {cap }}}{R_{\text {buck }}^{2}} \propto \frac{R_{0}{ }^{2}}{R_{\text {buck }}{ }^{2}}
$$

Hence

$$
\left(\frac{T}{R}\right)_{\text {buck }} \propto\left(\frac{R_{0}}{R_{\text {buck }}}\right)^{3}
$$

Thus, in this model a critical buckling ratio $\left(\frac{T}{R}\right)_{\text {buck }}$ is equivalent to a critical radius ratio $R_{0} / R_{\text {buck }}$. Comparing to eq 6 a critical radius ratio also corresponds to a critical film density. Consequently, the observed scaling of the buckling diameter can be accounted for: a constant ratio $\left(\frac{T}{R}\right)_{\text {buck }}$ is explained if we assume a constant Poisson ratio and a phase change at a critical interfacial density. The 3-dimensional Young's modulus that is measured at buckling is $\sim 10^{5} \mathrm{~N} \mathrm{~m}^{-2}\left(Y_{3 \mathrm{D}}=\frac{Y_{2 \mathrm{D}}}{T}\right.$, Table 1) which is consistent with the phase change being a gelation. It is well established that $\mathrm{CB}[8]$-cross-linked polymers gel at a few wt $\% .^{22,27}$

Liquid-to-gel phase transitions are dependent on the density of cross-links in the polymer network, with the Young's modulus proportional to the cross-link density. ${ }^{28,29}$ This justifies the assumption of a critical film density and implies that changing the degree of cross-linking should alter the buckling diameter. We believe that this accounts for the behavior observed in Figure 2a, where a smaller buckling diameter is observed with a lower proportion of the $\mathrm{CB}[8]$ supramolecular cross-linker. With a smaller number of crosslinks the material has to compress by a greater extent to reach the same cross-link density and so reaches a smaller diameter before the interfacial layer gels and buckles.

So far the trigger of shell buckling has been the focus of analysis. It is found that the buckling is triggered by a change in material properties as a result of a compression-induced phase change. However, the buckling process itself is also worth commenting on. While the compression prior to the phase change is spherical and isotropic, after the phase change the capsules buckle anisotropically and consistently around a central indent (Figure 1d). We believe that this is caused by stresses around a hoop at the oil-droplet-air interface. There have been several prior studies that focus on anisotropic film 
buckling and creating structured capsules. ${ }^{23,30,31}$ Using anisotropic forces at fluid interfaces may be another route toward creating such structures.

\section{CONCLUSIONS}

Fabricating microcapsules at the interface of microdroplets combines tunable functionality with straightforward and quantitative cargo encapsulation. However, previously crucial elements of microcapsule formation have not been well understood which has hindered rational capsule design.

Here the mechanism of microcapsule formation, and in particular the onset of buckling, was studied. The buckling diameter was found to scale with the cubic root of the material mass, independent of whether additional mass originated from a greater initial concentration or larger initial droplets. This scaling implies a constant thickness-to-radius ratio at buckling, $\left(\frac{T}{R}\right)_{\text {buck }}$. By measuring the film material properties, as well as by imaging the fluidity of the interface, it was inferred that the onset of buckling coincides with a liquid-to-solid phase transition. Specifically, here this phase transition is a gelation, as evidenced by the Young's modulus of the polymeric shell. Further, we show that such compression-induced interfacial phase changes can be quantitatively and simply described in terms of an increase in the density of the film associated with a Poisson ratio $0<\nu<0.5$. Many types of phase changes (including particle jamming) result from a density increase and so we expect this description to apply widely.

These results have several implications with respect to the design of capsules by this method. Greater wall thicknesses can be achieved by increasing the material concentration or, equivalently, the initial droplet size. Buckling behavior will be material dependent, bearing a strong relation to gelation (or other phase change) behavior.

\section{ASSOCIATED CONTENT}

\section{S Supporting Information}

The Supporting Information is available free of charge on the ACS Publications website at DOI: 10.1021/acs.langmuir.6b03011.

Derivation of eq 6; scaling of the buckling radius for a different polymer system; calculations relating to the host-guest equilibrium; kinetic experiments and AFM and interferometry measurements of the ratio $\left(\frac{T}{R}\right)_{\text {buck }}$ (PDF)

\section{AUTHOR INFORMATION}

\section{Corresponding Author}

*E-mail: ca26@cam.ac.uk.

\section{Notes}

The authors declare no competing financial interest.

Additional data related to this publication is available at the University of Cambridge data repository http://dx.doi.org/10. 17863/CAM.4910.

\section{ACKNOWLEDGMENTS}

P. Cicuta is acknowledged for support and discussions. A.S. and A.G. acknowledge the support of the EPSRC Cambridge NanoDTC (EP/L015978/1 and EP/G037221/1 respectively).

\section{REFERENCES}

(1) Martins, I. M.; Barreiro, M. F.; Coelho, M.; Rodrigues, A. E. Microencapsulation of Essential Oils with Biodegradable Polymeric Carriers for Cosmetic Applications. Chem. Eng. J. 2014, 245, 191-200.

(2) De Cock, L. J.; De Koker, S.; De Geest, B. G.; Grooten, J.; Vervaet, C.; Remon, J. P.; Sukhorukov, G. B.; Antipina, M. N. Polymeric Multilayer Capsules in Drug Delivery. Angew. Chem., Int. Ed. 2010, 49 (39), 6954-6973.

(3) Nazzaro, F.; Orlando, P.; Fratianni, F.; Coppola, R. Microencapsulation in Food Science and Biotechnology. Curr. Opin. Biotechnol. 2012, 23 (2), 182-186.

(4) Microencapsulation: Methods and Industrial Applications, 2nd ed.; Benita, S., Ed.; CRC Press: Boca Raton, FL, 2005.

(5) Shah, R. K.; Kim, J.-W.; Agresti, J. J.; Weitz, D. A.; Chu, L.-Y. Fabrication of Monodisperse Thermosensitive Microgels and Gel Capsules in Microfluidic Devices. Soft Matter 2008, 4 (12), 2303.

(6) Wang, J. T.; Wang, J.; Han, J. J. Fabrication of Advanced Particles and Particle-Based Materials Assisted by Droplet-Based Microfluidics. Small 2011, 7 (13), 1728-1754.

(7) Seiffert, S. Small but Smart: Sensitive Microgel Capsules. Angew. Chem., Int. Ed. 2013, 52 (44), 11462-11468.

(8) Sugiyama, Y.; Larsen, R. J.; Kim, J. W.; Weitz, D. A. Buckling and Crumpling of Drying Droplets of Colloid-Polymer Suspensions. Langmuir 2006, 22 (14), 6024-6030.

(9) Amstad, E.; Datta, S.; Weitz, D. A. The Microfluidic Post-Array Device: High Throughput Production of Single Emulsion Drops. Lab Chip 2014, 14 (4), 705-709.

(10) Zhang, J.; Coulston, R. J.; Jones, S. T.; Geng, J.; Scherman, O. A.; Abell, C. One-Step Fabrication of Supramolecular Microcapsules from Microfluidic Droplets. Science (Washington, DC, U. S.) 2012, 335 (6069), 690-694.

(11) Zheng, Y.; Yu, Z.; Parker, R. M.; Wu, Y.; Abell, C.; Scherman, O. A. Interfacial Assembly of Dendritic Microcapsules with Host-Guest Chemistry. Nat. Commun. 2014, 5, 5772.

(12) Parker, R. M.; Zhang, J.; Zheng, Y.; Coulston, R. J.; Smith, C. A.; Salmon, A. R.; Yu, Z.; Scherman, O. A.; Abell, C. ElectrostaticallyDirected Self-Assembly of Ultra-Thin Supramolecular Polymer Microcapsules. Adv. Funct. Mater. 2015, 25 (26), 4091-4100.

(13) Yu, Z.; Zhang, J.; Coulston, R. J.; Biedermann, F.; Liu, X.; Scherman, O. A.; Parker, R. M.; Abell, C. Supramolecular Hydrogel Microcapsules via cucurbit[8] uril Host-Guest Interactions with Triggered and UV-Controlled Molecular Permeability. Chem. Sci. 2015, 6 (8), 4929-4933.

(14) Quilliet, C. Numerical Deflation of Beach Balls with Various Poisson's Ratios: From Sphere to Bowl's Shape. Eur. Phys. J. E: Soft Matter Biol. Phys. 2012, 35 (6), 1-9.

(15) Tsapis, N.; Dufresne, E. R.; Sinha, S. S.; Riera, C. S.; Hutchinson, J. W.; Mahadevan, L.; Weitz, D. A. Onset of Buckling in Drying Droplets of Colloidal Suspensions. Phys. Rev. Lett. 2005, 94 (1), 18302 .

(16) Day, A.; Arnold, A. P.; Blanch, R. J.; Snushall, B. Controlling Factors in the Synthesis of Cucurbituril and Its Homologues. J. Org. Chem. 2001, 66 (24), 8094-8100.

(17) Kim, J.; Jung, I.; Kim, S.-Y.; Lee, E.; Kang, J.; Sakamoto, S.; Yamaguchi, K.; Kim, K. New Cucurbituril Homologues: Syntheses, Isolation, Characterization, and X-Ray Crystal Structures of Cucurbit[n] uril (N= 5, 7, and 8). J. Am. Chem. Soc. 2000, 122 (3), 540-541.

(18) Duffy, D. C.; McDonald, J. C.; Schueller, O. J.; Whitesides, G. M. Rapid Prototyping of Microfluidic Systems in Poly(dimethylsiloxane). Anal. Chem. 1998, 70 (23), 4974-4984.

(19) Ziegler, C.; Eychmuller, A. Seeded Growth Synthesis of Uniform Gold Nanoparticles with Diameters of 15-300 Nm. J. Phys. Chem. C 2011, 115 (11), 4502-4506.

(20) Knoche, S.; Vella, D.; Aumaitre, E.; Degen, P.; Rehage, H.; Cicuta, P.; Kierfeld, J. Elastometry of Deflated Capsules: Elastic Moduli from Shape and Wrinkle Analysis. Langmuir 2013, 29 (40), 12463-12471. 
(21) Kim, K.; Selvapalam, N.; Oh, D. H. Cucurbiturils: A New Family of Host Molecules. J. Inclusion Phenom. Mol. Recognit. Chem. 2004, 50 (1-2), 31-36.

(22) Appel, E. A.; Biedermann, F.; Rauwald, U.; Jones, S. T.; Zayed, J. M.; Scherman, O. A. Supramolecular Cross-Linked Networks via Host- Guest Complexation with Cucurbit[8]uril. J. Am. Chem. Soc. 2010, 132 (40), 14251-14260.

(23) Datta, S. S.; Kim, S. H.; Paulose, J.; Abbaspourrad, A.; Nelson, D. R; Weitz, D. A. Delayed Buckling and Guided Folding of Inhomogeneous Capsules. Phys. Rev. Lett. 2012, 109 (13), 134302.

(24) Jose, J.; Kamp, M.; Van Blaaderen, A.; Imhof, A. Unloading and Reloading Colloidal Microcapsules with Apolar Solutions by Controlled and Reversible Buckling. Langmuir 2014, 30 (9), 23852393.

(25) Erni, P.; Jerri, H. A.; Wong, K.; Parker, A. Interfacial Viscoelasticity Controls Buckling, Wrinkling and Arrest in Emulsion Drops Undergoing Mass Transfer. Soft Matter 2012, 8 (26), 69586967.

(26) Aumaitre, E.; Wongsuwarn, S.; Rossetti, D.; Hedges, N. D.; Cox, A. R.; Vella, D.; Cicuta, P. A Viscoelastic Regime in Dilute Hydrophobin Monolayers. Soft Matter 2012, 8 (4), 1175.

(27) Appel, E. A.; Loh, X. J.; Jones, S. T.; Dreiss, C. A.; Scherman, O. A. Sustained Release of Proteins from High Water Content Supramolecular Polymer Hydrogels. Biomaterials 2012, 33 (18), 4646-4652.

(28) Gardel, M. L.; Shin, J. H.; MacKintosh, F. C.; Mahadevan, L.; Matsudaira, P.; Weitz, D. A. Elastic Behavior of Cross-Linked and Bundled Actin Networks. Science (Washington, DC, U. S.) 2004, 304 (5675), 1301-1305.

(29) MacKintosh, F. C.; Käs, J.; Janmey, P. A. Elasticity of Semiflexible Biopolymer Networks. Phys. Rev. Lett. 1995, 75 (24), $4425-4428$.

(30) Shchepelina, O.; Kozlovskaya, V.; Kharlampieva, E.; Mao, W.; Alexeev, A.; Tsukruk, V. V. Anisotropic Micro- and Nano-Capsules. Macromol. Rapid Commun. 2010, 31 (23), 2041-2046.

(31) Jiang, C.; Singamaneni, S.; Merrick, E.; Tsukruk, V. V. Complex Buckling Instability Patterns of Nanomembranes with Encapsulated Gold Nanoparticle Arrays. Nano Lett. 2006, 6, 2254-2259. 